\title{
Presence probability of Hemiscorpius lepturus Peters, 1861 using maximum entropy approach in the western areas of Zagros Mountains, Iran
}

\author{
Ahmad Ali Hanafi-Bojd ${ }^{1}$, Mona Sharififard ${ }^{2,3}$, Elham Jahanifard ${ }^{2,3}$, Shahrokh Navidpour ${ }^{4}$ and Babak Vazirianzadeh ${ }^{2,3}$
}

1. Department of Medical Entomology and Vector Control, School of Public Health, Tehran University of Medical Sciences, Tehran, Iran; 2. Social Determinants of Health Research Center, Ahvaz Jundishapur University of Medical Sciences, Ahvaz, Iran; 3. Department of Medical Entomology and Vector Control, School of Public Health, Ahvaz Jundishapur University of Medical Sciences, Ahvaz, Iran; 4. Razi Reference Laboratory of Scorpion Research, Razi Vaccine and Serum Research Institute, Agricultural Research Education and Extension Organization, Karaj, Iran.

Corresponding author: Elham Jahanifard, e-mail: jahanifard-e@ajums.ac.ir

Co-authors: AAH: aahanafibojd@tums.ac.ir, MS: sharififard-m@ajums.ac.ir, SN: navid1038@hotmail.com, BV: babakvazir@yahoo.co.uk

Received: 01-10-2019, Accepted: 30-12-2019, Published online: 17-02-2020

doi: www.doi.org/10.14202/vetworld.2020.296-303 How to cite this article: Hanafi-Bojd AA, Sharififard M, Jahanifard E, Navidpour S, Vazirianzadeh B (2020) Presence probability of Hemiscorpius lepturus Peters, 1861 using maximum entropy approach in the western areas of Zagros Mountains, Iran, Veterinary World, 13(2):296-303.

\begin{abstract}
Aim: The purpose of this research was to use environmental variables for predicting the probability of Hemiscorpius lepturus existence in the provinces where situated in the west of the Zagros Mountains.

Materials and Methods: In this study, 64 occurrence records of the H. lepturus were extracted from the published documents available in electronic databases. MaxEnt model was used for predicting the ecological niches of this species. Normalized difference vegetation index (NDVI) and 19 climatic variables were used as the environmental variables affecting the distribution of this scorpion. The Jackknife test in the model was used to indicate the importance of variables to predict the probability of the presence of the studied species. The logistic threshold that was evaluated using a logistic regression algorithm showed the converting of the probability model into a binary model. The model was evaluated byarea under the curve (AUC). The probability presence map of this scorpion was then prepared in ArcGIS 10.5 Software.
\end{abstract}

Results: The results of the analysis showed that the most important environmental factor on the distribution of $H$. lepturus was the maximum temperature of the warmest month (Bio5) with a contribution rate of $43 \%$ and permutation importance of $8 \%$. The Jackknife test revealed that NDVI did not gain any value when it used independently in the model. The logistic threshold was reported 0.255 for the maximum test sensitivity plus specificity. The AUC of the model was 0.7698 , shows an acceptable value for model validity. Overall the hot spots for this toxic scorpion seem to be in Khuzestan, Lorestan, and Ilam Provinces of the studied area.

Conclusion: Regarding our findings, MaxEnt algorithm, in combination with geographic information system contributed to revealing the effects of environmental variables on the probability of $H$. lepturus presence in the west of Zagros Mountains. These visualized maps as a warning alarm can be helpful to policymakers for managing, controlling, and monitoring the scorpionism in high-risk areas.

Keywords: ecological niches, Hemiscorpius lepturus, Iran, MaxEnt, Zagros mountains.

\section{Introduction}

Scorpions are important medical arthropods that belong to the class of Arachnida and scorpionidae order that are active at night and are mainly found in hot and dry areas [1]. These animals are not living fossils due to various adaptations, including behavioral, physiological, and ecological which ensure their success during 450 million years ago [2]. The arthropods have a venomous sting and almost 1500 species of scorpions are known worldwide, about 30 of which are potentially dangerous to humans [3]. Nowadays,

Copyright: Hanafi-Bojd, et al. Open Access. This article is distributed under the terms of the Creative Commons Attribution 4.0 International License (http://creativecommons.org/licenses/ by/4.0/), which permits unrestricted use, distribution, and reproduction in any medium, provided you give appropriate credit to the original author(s) and the source, provide a link to the Creative Commons license, and indicate if changes were made. The Creative Commons Public Domain Dedication waiver (http:// creativecommons.org/publicdomain/zero/1.0/) applies to the data made available in this article, unless otherwise stated. scorpions are found in terrestrial habitat and Iran is a rich region for many species of dangerous scorpions due to climatic conditions [1]. There are three families of Scorpionidae, Hemiscorpiidae, and Buthidae in Iran that $86 \%$ of species is related to Buthidae [4]. Scorpion sting is one of the major health problems in Iran that has caused many deaths in the south and southwestern part of the country [5]. According to the incommunicable Disease Management Center of Ministry of Health and Medical Education, more than 50,000 stings are reported in the country. Although the most scorpionism and deaths occur in Khuzestan Province, it is reporting from other parts of the country as well [1]. The average of the incidence of scorpionism is about 59.5/100,000 population in Iran. Most cases of scorpion stings happened in Khuzestan Province with 541/100,000 population. Moreover, Hormozgan, Bushehr, and Ilam provinces with $15.9,127$, and $123 / 100,000$ are in the next position, 
respectively [6]. The incidence and severity of clinical signs of scorpion sting depend on the species of scorpion, the physiological conditions of the patient, the number of stings, the amount of toxin injected, the age, weight, and health of the patient. Clinical effects can range from simple localized pain to severe systemic reactions that sometimes lead to death [7].

Anepidemiologicalsurvey ofenvenomationshowed that there are at least seven medically important scorpion species in Iran. One of them is Hemiscorpius lepturus (Gadim) that belongs to the Hemiscorpiidae family [6]. It worth noting that one genus (Hemiscorpius) and seven species (Hemiscorpius acanthocercus, Hemiscorpius enischnochela, Hemiscorpius gaillardia, Hemiscorpius lepturus, Hemiscorpius persicus, Hemiscorpius kashkayi, and Hemiscorpius shahii) of this family reported from Iran $[8,9]$. The family Hemiscorpiidae is distributed in East Africa, the Arabian Peninsula, Iraq, and Iran [10]. Moreover, H. lepturus as a non-digger scorpion distributed in 15 of 31 provinces in the country such as Khuzestan, Hormozgan, Bushehr, Kerman, Fars, Kohgiluyeh and Boyer Ahmad, Chaharmahal and Bakhtiari, Ilam, and Lorestan [8,11-21]. The color of this scorpion is light yellow to brown. While, moveable and fixed fingers of pedipalps are reddish. Sexual dimorphism was observed in this species, such that males have significantly longer metasoma than females [22]. The scorpion has a longer carapace than wide with stout and bulky pedipalps. The vesicle of telson is ovoid, globular, and bulky with fine and thin stings. The size of males can vary greatly from $52 \mathrm{~mm}$ up to $85 \mathrm{~mm}$ body length; females display less variation in size. Furthermore, 8-11 teeth and 14-16 teeth exist in pectines of females and males, respectively [23]. The venom of $H$. lepturus is composed of cytotoxins and hemotoxins. And also, it had a greater effect on erythrocyte hemolysis than other important species, Androctonus crassicauda, in the area. Its symptoms can include hematuria, acute and secondary renal failure, skin rashes, blistering, and necrosis of scorpion sting and surrounding tissues and very little pain such as itching or bee stings [8]. The severe hemoglobinuria observed in $95 \%$ of $H$. lepturus victims. Previous studies showed that the highest fatality rate in Khuzestan Province was related to envenomation by $H$. lepturus. This scorpion is responsible for $10-25 \%$ of the scorpion sting in the warm seasons and throughout the winter. Moreover, $24.9 \%$ of total scorpionism in Khuzestan Province is due to Gadim [24]. Children are at risk group of $H$. lepturus sting with high mortality among them [25]. The emergency injection of antivenom is essential in $H$. lepturus envenomation due to hemolysis and, consequently, acute renal failure in the first $24 \mathrm{~h}$ after scorpion sting [26]. Intramuscular injection of antivenoms is ineffective in neutralizing the action of venom [27]. While the intramuscular injection route of antivenom is useful, only under $2 \mathrm{~h}$ following $H$. lepturus sting [28].

Species distribution models are analytical or statistical algorithms that can predict the actual and potential species distribution by linking among field observations and environmental factors [29]. One of the methods for determining the habitat is the use of predictive models of dispersal that their base is quantifying the relationship between species and different environmental variables [30]. Models based on the concept of ecological niche modeling provide good information on possible species distribution when there is insufficient data and also can be used in species conservation planning [29]. One of these methods is the maximum entropy or MaxEnt, which is used to represent species distribution [31]. The MaxEnt model is a machine learning based on the maximum disorder that is used to predict the presence when data are not available in the region. This method estimates the probability of the presence of a species based on the constraints obtained from existing data [32]. MaxEnt is a program for modeling the species distribution of the collected samples. Furthermore, this method is used for various aims including predicting the potential distribution of species, phyloclimatic studies, understanding environmental and ecological correlates of species occurrences [33]. Ecological and climatic variables in analyses based geographic information system (GIS) reported as determining the species boundaries [34]. GIS in combination with ecological niche modeling was applied to preparing the risk maps and predict the distribution of some medically important insects and arthropods [29, 35-38].

The purpose of this study was to use environmental factors in combination with the MaxEnt model for predicting the probability of $H$. lepturus presence as the dependent variable in the provinces in the west of the Zagros Mountains in Iran. The model prediction ability was evaluated by the AUC value. Furthermore, we can determine the contribution value of each environmental variable in the distribution of the scorpion in study areas. The present study is able to complete zoo-geographical data to design the management strategies based on ecological niche modeling and to prepare the visualize map for monitoring the scorpionism in high-risk regions.

\section{Materials and Methods}

\section{Ethical approval}

All articles that used in the present study were referenced in the paper. Furthermore, this study was approved by the Ethical Committee on December 4, 2016, at Ahvaz Jundishapur University of Medical Sciences, Iran (No. IR.AJUMS.REC.1395.538).

\section{Study area}

Iran, a country in the southwest of Asia and the Middle East, with $32.4279^{\circ} \mathrm{N}$ and $53.6880^{\circ} \mathrm{E}$ covers an area of 1,648,195 $\mathrm{km}^{2}$. According to data from Statistical Center of Iran, it ranks $18^{\text {th }}$ in the World with 79,926,270 human population. It is bordered by Azerbaijan, Armenia, and Turkmenistan to the north, Afghanistan, and Pakistan to the east, and Turkey and Iraq to the west. It also limited to the Caspian Sea from the north, and the Persian Gulf and Oman Sea 
to the south, which are the first two major oil and gas extraction sites in the world.

The Zagros Mountains are a long mountain range in Iran, Iraq, and Southeast Turkey. This mountain range has a total length of $1600 \mathrm{~km}$. The Zagros mountain range begins in Northwest Iran and roughly follows Iran's western border, while covering much of Southeast Turkey and Northeast Iraq. Moreover, the provinces located west of Zagros Mountains in Iran are Kermanshah, Chaharmahal andBakhtiari, Bushehr, Fars, Khouzestan, Ilam, Lorestan, Kohgiluyeh dnaBoyer-Ahmad, and Hormozgan (Figure-1).

\section{Data collection}

The occurrence record of $H$. lepturus was extracted from the published documents by searching on databases such as Google Scholar, PubMed, Scopus, Iran Medex, Elsevier, and Scientific Information Database. Fauna, biodiversity, scorpion, species composition, and Iran were used as keywords for the search strategy. Researches that had been down in Kermanshah, Chaharmahal and Bakhtiari, Bushehr, Fars, Khuzestan, Ilam, Lorestan, Kohgiluyeh and Boyer Ahmad, and Hormozgan Provinces and collected H. lepturus scorpion were included in the study. The exclusion criteria were regarded as other species except for H. lepturus. Moreover, latitude and longitude of sampling locations were used for creating the species databank. All data designed in Microsoft Excel and its table added to ArcGIS 10.5. Then, shapefile of $H$. lepturus prepared in ArcMap application and it was used for showing geographical distribution and modeling of the presence chance of $H$. lepturus.

\section{MaxEnt modeling}

MaxEnt is suitable for modeling species' geographic distributions with available information, including climatic data and land cover [32]. Environmental variables include 19 items (Bio1: Annual Mean Temperature $\left({ }^{\circ} \mathrm{C}\right)$; Bio2: Mean diurnal range (mean of monthly [max temp-min temp]) $\left({ }^{\circ} \mathrm{C}\right)$; Bio3: Isothermality (Bio2/Bio7) $(\times 100)$; Bio4: Temperature

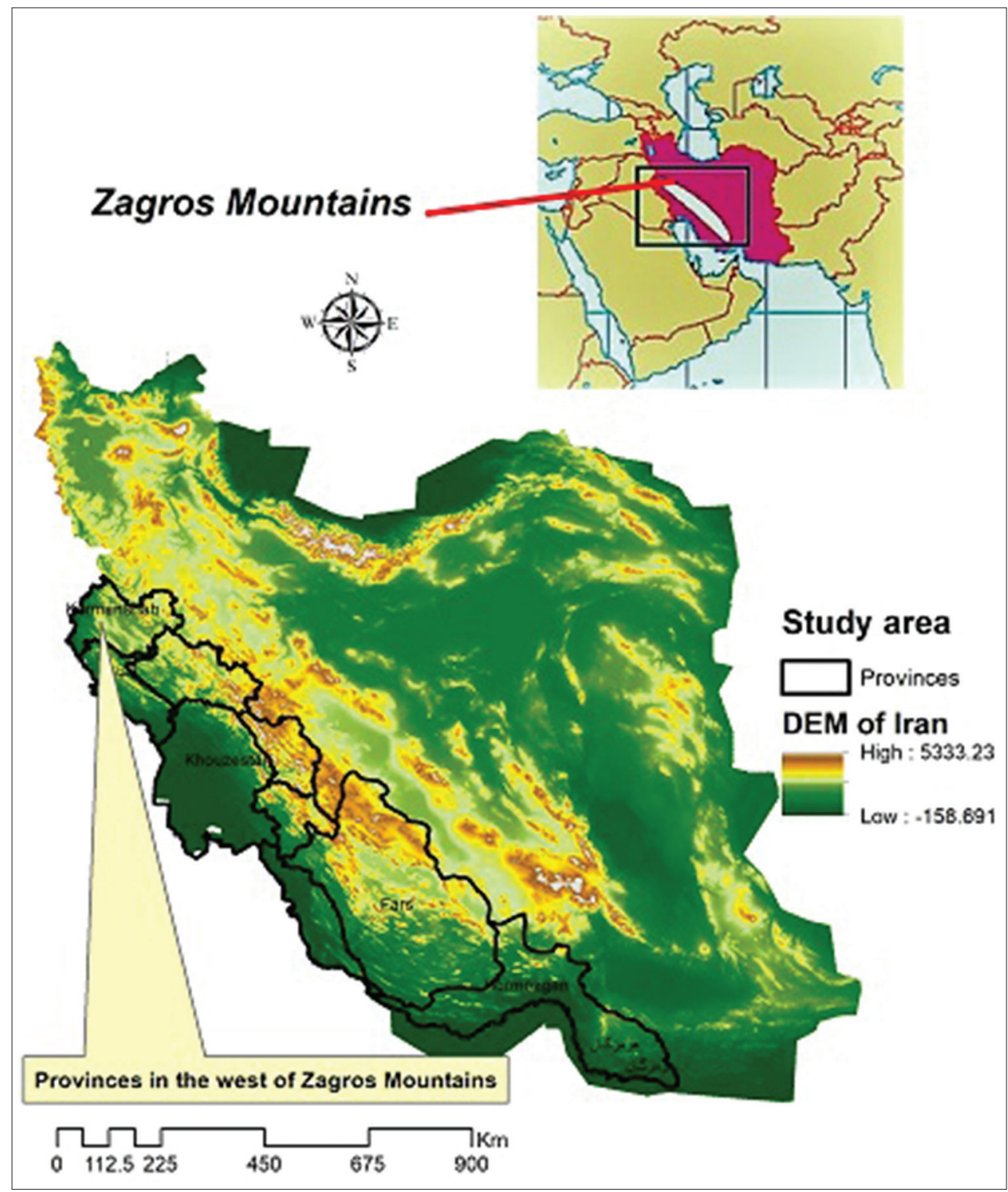

Figure-1: Study areas in the west of Zagros Mountains [Map prepared by EJ]. 
seasonality (standard deviation $\times 100$ ); Bio5: Max temperature of warmest month $\left({ }^{\circ} \mathrm{C}\right)$; Bio6: Min temperature of coldest month $\left({ }^{\circ} \mathrm{C}\right)$; Bio7: Temperature annual range (Bio5-Bio6) temperature of wettest quarter $\left({ }^{\circ} \mathrm{C}\right.$ ); Bio8: Mean temperature of wettest quarter $\left({ }^{\circ} \mathrm{C}\right)$; Bio9: Mean temperature of driest quarter $\left({ }^{\circ} \mathrm{C}\right)$; Bio10: Mean temperature of warmest quarter $\left({ }^{\circ} \mathrm{C}\right)$; Bio11: Mean temperature of coldest quarter $\left({ }^{\circ} \mathrm{C}\right)$; Bio12: Annual precipitation (mm); Bio13: Precipitation of wettest month (mm); Bio14: Precipitation of driest month (mm); Bio15: Seasonal precipitation (coefficient of variation); Bio16: Precipitation of wettest quarter (mm); Bio17: Precipitation of driest quarter (mm); Bio18: Precipitation of warmest quarter $(\mathrm{mm})$; and Bio19: Precipitation of coldest quarter) in raster format with spatial resolution of $1 \mathrm{~km}^{2}$ were downloaded from the WorldClim database (http://www.worldclim. org/current). Normalized difference vegetation index (NDVI) gained from the image of moderate resolution imaging spectroradiometer satellite in 2014. Eighty percent of the occurrence recodes of H. lepturus were randomly used by the software for training the model and the remaining $20 \%$ for the test.

The logistic threshold that was evaluated using a logistic regression algorithm showed the converting of the probability model into a binary model.

The Jackknife Estimation method used to indicate the association of all variables to predict species distribution. The accuracy of modeling geographic distribution of Gadim was evaluated by receiver operating characteristic (ROC) curve. The area under an ROC curve (AUC) is between maximum 1 and minimum 0 [39]. An excellent model has AUC near to the one which means it has a good measure of separability. Moreover, AUC near to zero means it has the worst measure of separability and the model is poor. The model performance regarding AUC was classified into failing, poor, fair, good, and excellent in the range of $0.5-0.6,0.6-0.7,0.7-0.8,0.8-0.9$, and $0.9-1$, respectively [40].

\section{Results}

The ecological niche model, MaxEnt, predicted the presence probability of $H$. lepturus in the west of Zagros Mountains. Regarding previous studies, 64 points were used for modeling this species, 48 records for training, and 16 for testing the model.

The relative contributions of all environmental variables were estimated in this model. The results of the analysis showed that the most important environmental factors in the distribution of $H$. lepturus were the maximum temperature of the warmest month (Bio5) contributed $43 \%$ with $8 \%$ permutation importance. Furthermore, Bio6 (min temperature of the coldest month), Bio8 (mean temperature of the wettest quarter), and Bio16 (precipitation of wettest quarter) did not contribute to MaxEnt algorithm.

The results of the Jackknife test related to variable important are indicated in Figure-2. The climatic variable of bio5 had the most useful information in predicting $H$. lepturus. Mean diurnal range $\left({ }^{\circ} \mathrm{C}\right)$, mean temperature of the driest quarter $\left({ }^{\circ} \mathrm{C}\right)$, and mean temperature of the warmest quarter $\left({ }^{\circ} \mathrm{C}\right)$ had moderate value when they used independently.

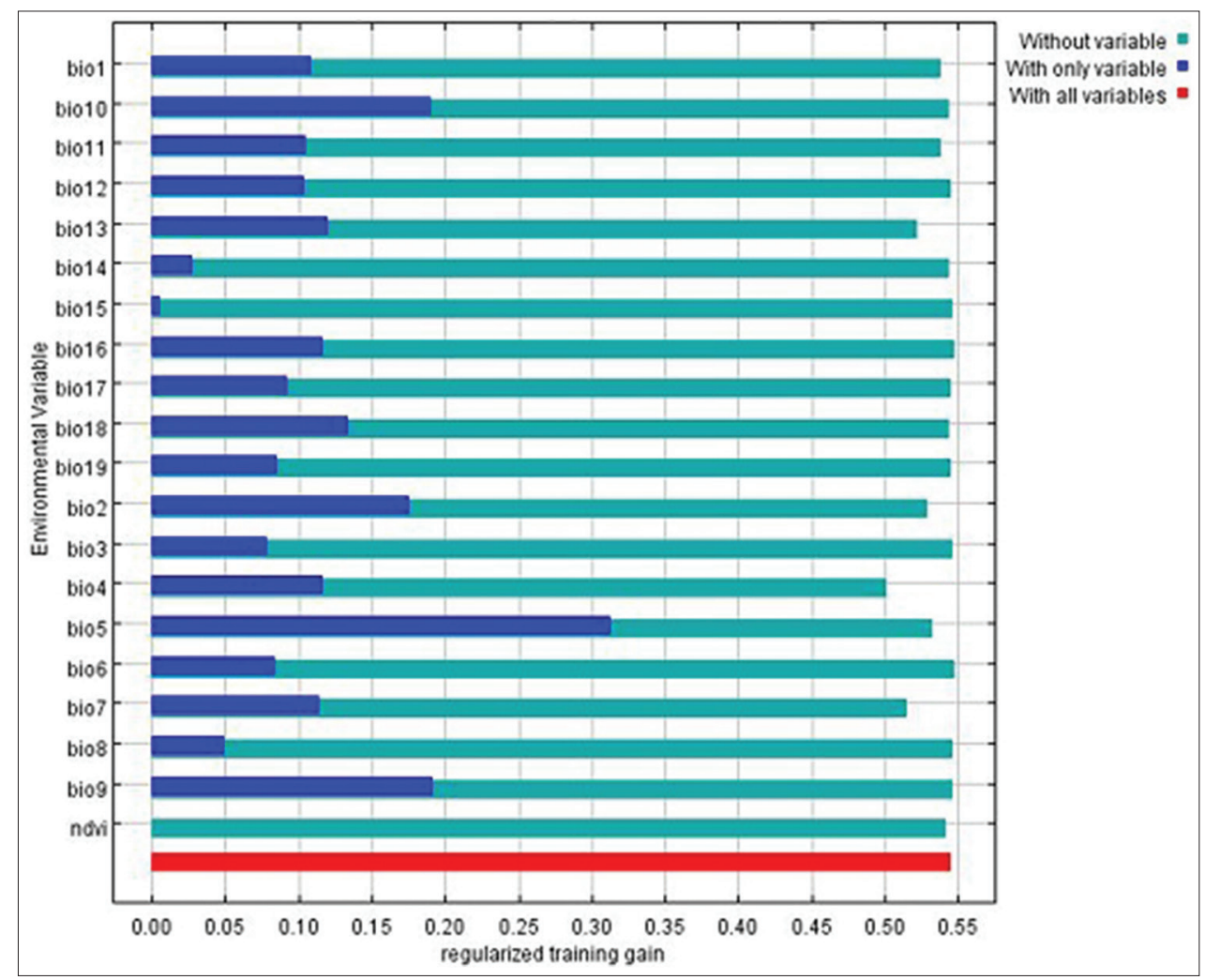

Figure-2: Jackknife of regularized training gain for Hemiscorpius lepturus in the west of Zagros Mountains in Iran. 
Temperature seasonality $\left({ }^{\circ} \mathrm{C}\right)$ is an environmental variable that has the most information toward other variables. With missing bio4, the estimation of environmental variables in modeling is reduced. The NDVI did not gain any value when it used independently in the model.

Geographical distribution and collecting sites of the species in study areas are indicated in Figure-3a. There was no valuable and published data on the presence of H. lepturus in Kermanshah Province but it is reported from other provinces in the west of Zagros Mountain. Figure- $3 b$ is a representation of the MaxEnt model for $H$. lepturus that the better predicted conditions are indicated by the warmer colors. The presence probability of the species by percentage is shown in Figure- 4 in five classes $(0-20,21-40,41-60,61-80$, and more than 80). In other words, the presence chance of $H$. lepturus increases in the last classes that are equal to the warmest color. The most probability of the scorpion existence is predicted in Khuzestan Province and followed by Lorestan and Ilam provinces.

The binomial probabilities were calculated exactly if the number of test samples was at most 25 . The logistic threshold was reported 0.255 for the maximum test sensitivity plus specificity. Furthermore, thresholds resulting from MaxEnt for maximum training sensitivity plus specificity, equal training sensitivity and specificity and balance training omission, and

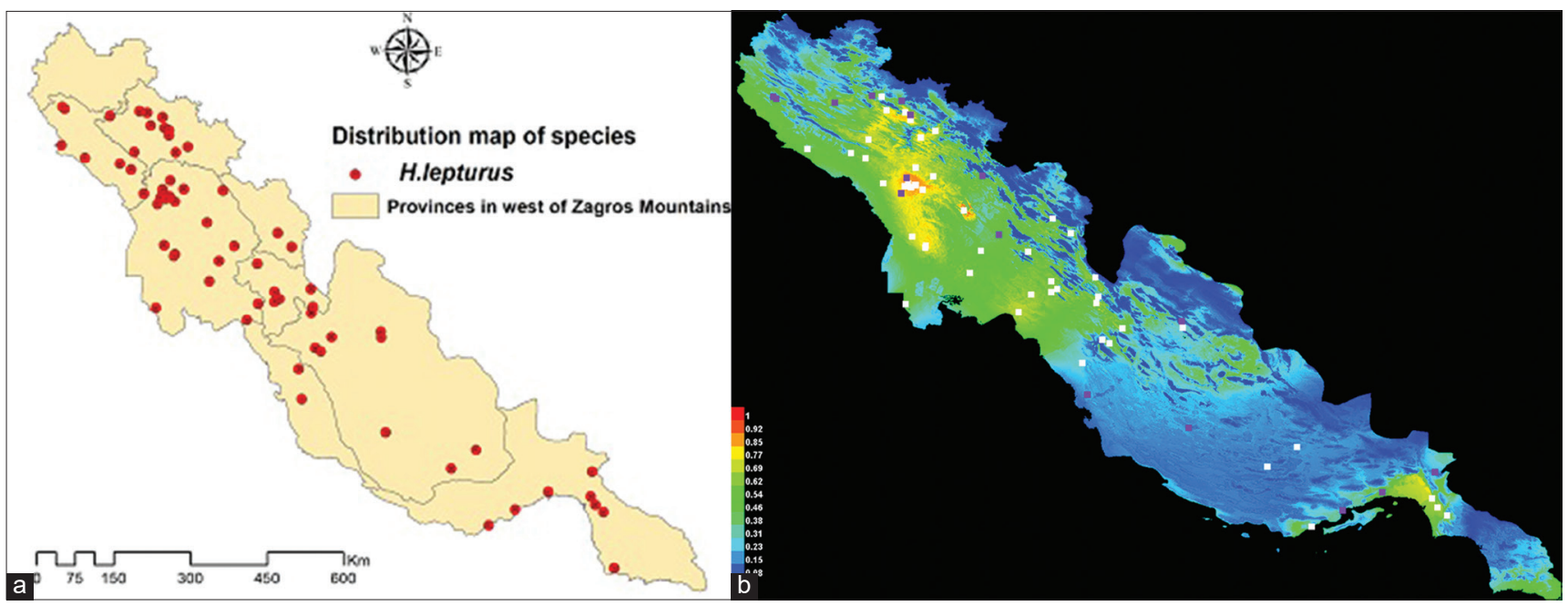

Figure-3: Spatial distribution (a) and prediction (b) of Hemiscorpius lepturus in the west of Zagros Mountains in Iran.

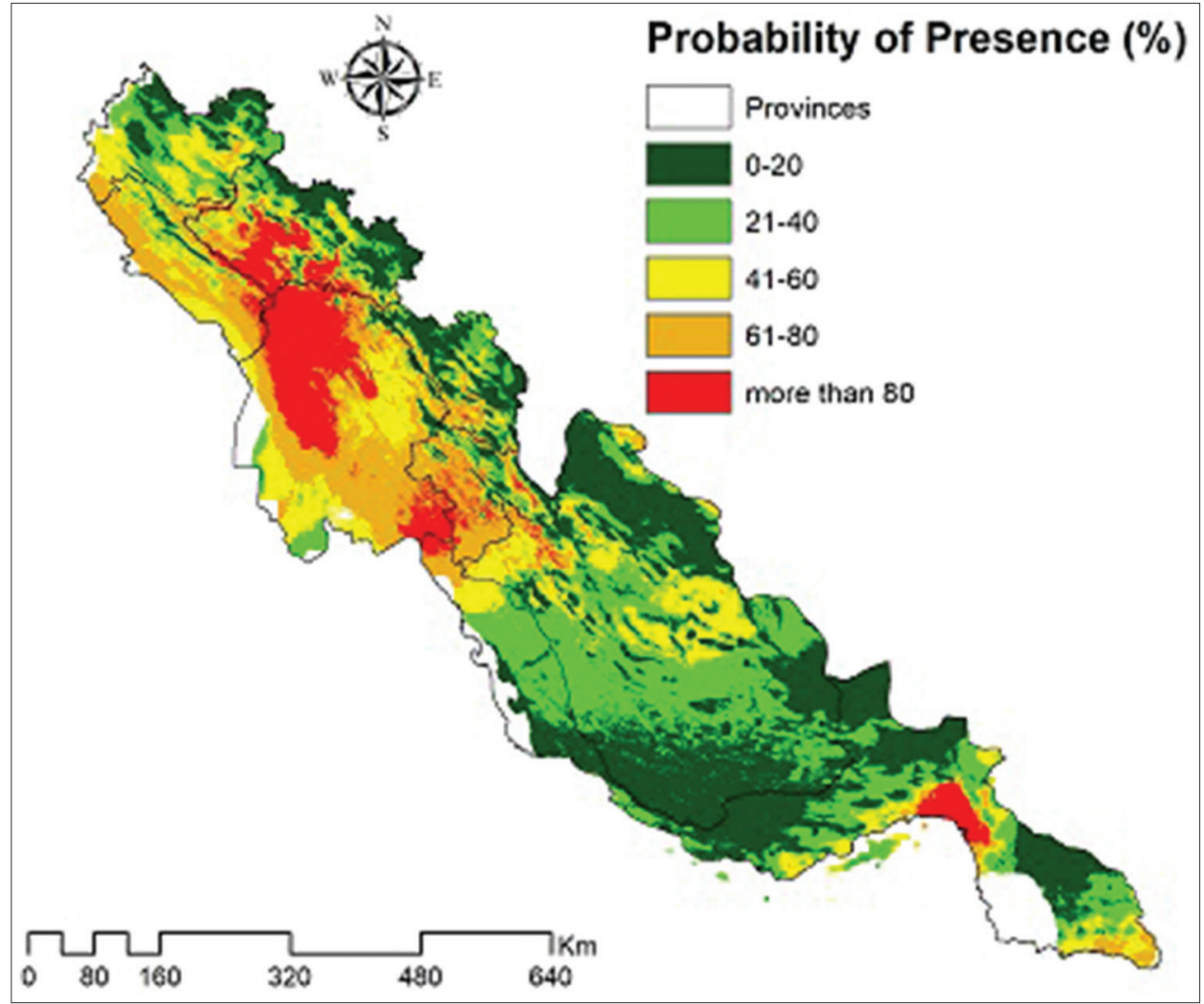

Figure-4: Presence probability of Hemiscorpius lepturus in the west of Zagros Mountains. 


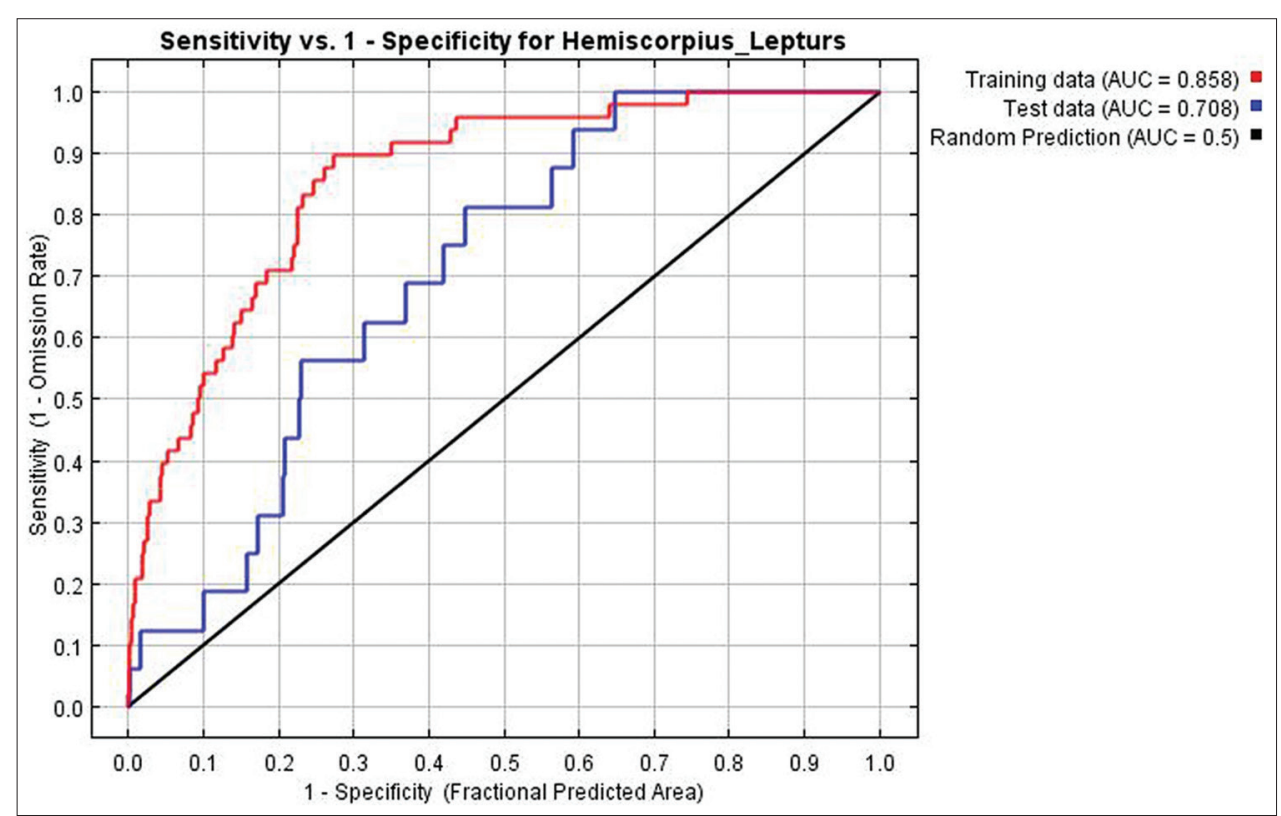

Figure-5: Model suitability using the AUC for training and test data.

predicted area and threshold value were calculated $0.422,0.467$, and 0.131 , respectively.

The area under the curve (AUC) for training and test data was 0.858 and 0.708 , respectively (Figure-5). When AUC is 0.7 , it means there is $70 \%$ chance that the model will be able to distinguish between positive class and negative class. The model performance was in the range of 0.7-0.8 and it was evaluated fairly.

\section{Discussion}

The majority of researches in Iran has focused on the fauna, diversity, and spatial distribution of scorpions, but the present research is able to predict this species in areas where no data are available. Regarding this study, the geographical distribution of $H$. lepturus focused especially on the provinces in the west and southwest of Iran, where the climate was hot and humid in most of them. Epidemiological studies showed that the major envenomation of $H$. lepturus happened in the southwest of the country [8]. Despite, reports of this species in Isfahan and Semnan Provinces [8], the highest distribution of this species is observed in the west of Zagros Mountains, which may be due to these mountains as a natural barrier to its geographical distribution.

The mean temperature of the wettest quarter of the year, precipitation of the coldest quarter of the year, and precipitation of the warmest quarter of the year were the most important variables that contributed to the distribution of Mesobuthus eupeus. Moreover, precipitation of the warmest quarter of the year as a variable had the highest contribution on Mesobuthusphillipsii distribution [30]. Lira et al. [41] showed the frequency of Tityus pusillus depends on environmental factors. Furthermore, another study confirmed the abundance fauna of the animal influences by response to the environmental condition [42].
Furthermore, El Hidan et al. [43] reported some species such as Androctonus amoreuxi, Androctonus liouvillei, Buthus mariefranceae, Buthus draa, Buthus boumalenii, and Buthus bonito adapted to Saharan bioclimate with rainfall $<150 \mathrm{~mm}$ and hot temperature. Our result showed that the relative contributions of the environmental variable were various from the previous study and it seems that the contributing factors are specialized for every species.

Our results presented that NDVI did not gain any value in predicting the presence of the species when it used independently in the model. Scorpio maurus indicated a negative correlation with vegetation cover [44]. H. lepturus as a lithophilous species lives in laminated rocks and its morphotype is affected by the special habitat [15] which seems to indicate that this variable is not involved in the distribution of this scorpion.

The distribution of scorpion species is associated mainly with climatic factors such as temperature, precipitation, and the nature of substratum such as soil texture and hardness, the thickness, and the amount of the stones [43]. Furthermore, rainfall, relative humidity, and temperature were known as the most dominant variables in species distribution, just like that Buthus malhommei and Buthus elmoutaouakili were frequently observed in the areas with low rainfall in the range of $150 \mathrm{~mm}-350 \mathrm{~mm}$ and high temperature [43].

Temperature variables were a good predictor to the distribution of Chibchea salta by maximum entropy [45]. El Hidan et al. [43] found that the environment has a significant role to show the presence of Androctonus Genus species. Moreover, environmental factors, including topography and climate, are two important variables that can affect on envenomation cases and frequency of venomous animals. However, they found the strongest relationship between the 
ranges of specific environmental factors with scorpion species. Some climatic circumstances, such as precipitation of coldest and warmest quarter, influenced Androctonus mauritanicus. However, the mean diurnal range, slope, wettest quarter precipitation, and warmest quarter were associated with A. liouvillei distribution. While $A$. amoreuxi was related to the mean temperature of the driest quarter, mean diurnal range, and slope [46]. In this study, according to the MaxEnt modeling, the important climatic factor in the presence of $H$. lepturus was the maximum temperature of the warmest month. It shows that it tends to live in warm areas that such bio-climates have been provided for the living of this scorpion in south and southwest of Iran.

The area under an ROC curve was calculated $0.950 \pm 0.025, \quad 0.988 \pm 0.006$, and $0.969 \pm 0.007$ for A. amoreuxi, A. liouvillei, and A. mauritanicus, respectively [46]. Brites-Neto and Duarte [38] reported AUC $=0.7698 \pm 0.0533$ for Tityus serrulatus and pointed to high predictive success. The results of this study presented that AUC for test data was $0.708 \pm 0.048$ which was evaluated fair by Swets' classification. The possibility map of $H$. lepturus can use as recognizing the people at risk and prevention of them from scorpion envenomation. Our result reflects that the accuracy of $H$. lepturus modeling was evaluated fairly. However, the most probability of the species presence predicted in Khuzestan Province and followed by Lorestan and Ilam, where the main habitats for the scorpion were consecutive years due to suitable climates and environment [8]. Epidemiological studies showed that H. lepturus was a principal cause of $10-15 \%$ of all hospital referred scorpion envenomation [8].

The strong point of this study is determining the appropriate species distribution only with presence data and recognizing absence data that are rarely available or reliable. Conclusions and interpretation of the results depend on the selected factors for analysis that can regard as weak points of the research. In our study, MaxEnt model was used to determine the distribution of $H$. lepturus by 19 environmental variables that show the limitation of our prediction to the validity of data.

\section{Conclusion}

Regarding our findings, the MaxEnt algorithm in combination with GIS contributed to revealing the effects of environmental variables on the probability of $H$. lepturus presence in the west of Zagros Mountains. Furthermore, both tools are able to produce the risk maps of the species stings where its envenomation is a serious public health problem. Considering the importance and distribution of this species in hot areas such as Khuzestan Province, preparing the scorpion envenomation risk map is possible by adding the demographical variables and interventional activities in ArcGIS 10.5. Based on our prediction results, we suggest using the visualized maps as a warning alarm as an appropriate tool to policymakers to manage, control, and monitor the scorpion stings in high-risk areas. Furthermore, raising the awareness, knowledge, and practice of exposed people to the scorpion will decrease scorpionism.

\section{Authors' Contributions}

MS, SN, and BV collected data, AAH analyzed data by MaxEnt and ArcGIS. EJ prepared the article. All authors participated in editing of the manuscript. All authors read and approved the manuscript.

\section{Acknowledgments}

This study was financially supported by Social Determinants of Health Research Center, Ahvaz Jundishapur University of Medical Sciences, Ahvaz, Iran with Project No. SDH-9515.

\section{Competing Interests}

The authors declare that they have no competing interests.

\section{Publisher's Note}

Veterinary World remains neutral with regard to jurisdictional claims in published map and institutional affiliation.

\section{References}

1. Dehghani, R., Rafinejad, J., Fathi, B., Shahi, M.P., Jazayeri, M. and Hashemi, A. (2017) A retrospective study on scropionism in Iran (2002-2011). J. Arthropod. Borne Dis., 11(2): 194-203.

2. Lourenço, W.R. (2015) Scorpion diversity and distribution; past and present patterns. In: Gopalakrishnakone, P., Possani, L.D., Schwartz, F. and Vega, R., editors. Scorpion Venoms, Toxinology. Springer+Business Media Dordrecht, Netherlands. p3-23.

3. Chippaux, J.P. (2012) Emerging options for the management of scorpions Stings. Drug Des. Dev. Therapy., 6: 165-173.

4. Dehghani, R. and Kassiri, H. (2018) A checklist of scorpions in Iran (by 2017). Asian J. Pharm., 12(3): S880-S887.

5. Shahbazzadeh, D., Amirkhani, A., Djadid, N.D., Bigdeli, S., Akbari, A., Ahari, H., Amini, H. and Dehghani, R. (2009) Epidemiological and clinical survey of scorpionism in Khuzestan province, Iran. Toxicon, 53(4): 454-459.

6. Rafizadeh, S., Rafinejad, J. and Rassi, Y. (2013) Epidemiology of scorpionism in Iran during 2009. $J$. Arthropod. Borne Dis., 7(1): 66-70.

7. Ryan, N., Buckley, N., Graudins, A. (2017) Treatments for latrodectism a systematic review on their clinical effectiveness. Toxins, 9(4): 148-168.

8. Dehghani, R., Kamiabi, F. and Mohammadi, M. (2018) Scorpionism by Hemiscorpius spp. in Iran: A review. J. Venom. Anim. Toxins incl. Trop. Dis., 24(8): 1-10.

9. Kovařík, F., Navidpour, S. and Soleglad, M.E. (2017) Hemiscorpius shahii sp. n. from Iran (Scorpiones: Hemiscorpiidae). Euscorpius., 2017(249): 1-9.

10. Soleglad, M.E., Fet, V. and Kovaŕík, F. (2005) The systematic position of the scorpion genera Heteroscorpion Birula, 1903 and Urodacus Peters, 1861 (Scorpiones: Scorpionoidea). Euscorpius, 2005(20): 1-38.

11. Navidpour, S., Kovarik, F., Soleglad, M.E. and Fet, V. (2008a) Scorpions of Iran (Arachnida, Scorpiones). Part I. Khoozestan province. Euscorpius, 2008(65): 1-8.

12. Navidpour, S., Fet, V., Kovarik, F. and Soleglad, M.E. 
(2008b) Scorpions of Iran (Arachnida, Scorpiones). Part II. Ilam province. Euscorpius, 2008(69): 1-31.

13. Navidpour, S., Kovarik, F., Soleglad, M.E. and Fet, V. (2008c) Scorpions of Iran (Arachnida, Scorpiones). Part IV. Kohgilouyeh and Boyer Ahmad province. Euscorpius, 74(170): 1-26.

14. Navidpour, S., Soleglad, M.E., Fet, V. and Kovarik, F. (2008d) Scorpions of Iran (Arachnida, Scorpiones). Part II. Bushehr province. Euscorpius, 2008(67): 1-35.

15. Navidpour, S., Nayebzadeh, H.H., Soleglad, M.E., Fet, V., Kovarik, F. and Kayedi, M.H. (2010) Scorpions of Iran (Arachnida: Scorpiones). Part VI. Lorestan province. Euscorpius, 99: 1-25.

16. Navidpour, S., Ezatkhan, M., Kovarik, F., Soleglad, M.E. and Fet, V. (2011) Scorpions of Iran (Arachnida: Scorpiones). Part VII. Kerman province. Euscorpius, 2011(131): 1-34.

17. Navidpour, S., Fet, V., Kovarik, F. and Soleglad, M.E. (2012) Scorpions of Iran (Arachnida, Scorpiones). Part VIII. Fars province. Euscorpius, 2012(139): 1-31.

18. Navidpour, S., Soleglad, M.E., Fet, V. and Kovarik, F. (2013) Scorpions of Iran (Arachnida, Scorpiones). Part IX. Hormozgan province, with a description of Odontobuthus tavighiae sp.n. (Buthidae). Euscorpius, 2013(170): 1-31.

19. Pirali-Kheirabadi, K., Navidpour, S., Fet, V., Kovarik, F. and Soleglad, M.E. (2009) Scorpions of Iran (Arachnida, Scorpiones). Part V. Chahar Mahal and Bakhtiyari province. Euscorpius, 2009(78): 1-25.

20. Sharifinia, N., Gowhari, I., Hoseiny-Rad, M. and Aivazi, A.A. (2017) Fauna and geographical distribution of scorpions in Ilam province, South-Western Iran. $J$. Arthropod. Borne Dis., 11(2): 242-248.

21. Haghi, F.M. and Dehghani, R. (2017) A review of scorpions reported in Iran. J. Mazandaran Univ. Med. Sci., 27(151): 213-226.

22. Jalali, A. and Rahim, F. (2014) Epidemiology review of scorpion envenomation in Iran. Iran. J. Pham. Res., 13(3): 743-756.

23. Monod, L. and Lourenco, W.R. (2005) Hemiscorpiidae (Scorpiones) from Iran, with descriptions of two new species and notes on biogeography and phylogenetic relationships. Rev. Suisse Zool., 112(4): 869-942.

24. Shahi, M., Dehghani, R. and Fathi, B. (2012) Scorpion sting in Iran: A review. Toxicon, 60(5): 919-933.

25. Chippaux, J.P. and Goyffon, M. (2008) Epidemiology of scorpionism: A global appraisal. Atca Trop., 107(2): 71-79.

26. Azizi, K., Akbarzadeh, A. and Ghobadifar, M.A. (2016) Yellow scorpion bite: Ignore or take it seriously? Iran. Red. Crescent Med. J., 18(12): e28942.

27. Mirakabadi, A.Z. (2013) Hemiscorpius lepturus envenomation: Manifestations and management with specific antivenom. Arch. Razi Inst., 68(2): 91-99.

28. Jalali, A., Pipelzadeh, M., Sayedian, R. and Rowan, E.G. (2010) A review of epidemiological, clinical and in vitro physiological studies of envenomation by the scorpion Hemiscorpius lepturus (Hemiscorpiidae) in Iran article. Toxicon, 55(2-3): 173-179.

29. Shi, C.M., Liang, H.B., Altanchimeg, D., Chuluunjav, C. and Zhang, D.X. (2015) Climatic niche defines geographical distribution of Mesobuthus eupeus mongolicus (Scorpiones: Buthidae) in Gobi desert. Zool. Syst., 40(3): 339-348.

30. Guisan, A. and Thuiller, W. (2005) Predicting species distribution: offering more than simple habitat models. Ecol. Lett., 8(9): 993-1009.
31. Farhadinia, M.S., Akbari, H., Mousavi, S.J., Eslami, M., Azizi, M., Shokouhi, J., Gholikhani N. and HosseiniZavarei, F. (2013) Exceptionally long movements of the Asiatic cheetah Acinonyx jubatus venatias across multiple arid reserves in central Iran. Orxy, 47(3): 427-430.

32. Phillips, S.J. and Dadik, M. (2008) Modelling of species distributions with MaxEnt: New extensions and a comprehensive evaluation. Ecography, 31(2): 161-175.

33. Elith, J., Phillips, S.J., Hastie, T., Dudík, M., Chee, Y.E. and Yates, C.J. (2011) A statistical explanation of MaxEnt for ecologists. Divers Distrib., 17(1): 43-57.

34. Raxworthy, C.J., Ingram, C.M., Rabibisoa, N. and Pearson, R.G. (2007) Applications of ecological niche modeling for species delimitation: A review and empirical evaluation using day geckos (Phelsuma) from Madagascar. Syst. Biol., 56(6): 907-923.

35. Mirshamsi, O. (2013) Ecological niche modeling of two scorpion species Mesobuthus eupeus (CL Koch, 1839) and M. phillipsii (Pocock, 1889) from the Iranian Plateau and Zagros region (Arachnida: Scorpiones). Euscorpius, 154:1-10

36. Hanafi-Bojd, A.A., Rassi, Y., Yaghoobi-Ershadi, M.R., Haghdoost, A.A., Akhavan, A.A., Charrahy, Z. and Karimi, A. (2015) Predicted distribution of visceral leishmaniasis vectors (Diptera: Psychodidae; Phlebotominae) in Iran: A niche model study. Zoonoses Public Health, 62(8): 644-654.

37. Hanafi-Bojd, A.A., Sedaghat, M.M., Vatandoost, H., AzariHamidian, S. and Pakdad, K. (2018) Predicting environmentally suitable areas for Anopheles superpictus Grassi (sl), Anopheles maculipennis Meigen (sl.) and Anopheles sacharovi Favre (Diptera: Culicidae) in Iran. Parasite Vector, 11(1): 382-394.

38. Brites-Neto, J. and Duarte, K.M. (2015) Modeling of spatial distribution for scorpions of medical importance in the São Paulo State, Brazil. Vet. World, 8(7): 823-830.

39. Pearce, J. and Ferrier, S. (2000) An evaluation of alternative algorithms for fitting species distribution models using logistic regression. Ecol. Model., 128(2-3): 147-127.

40. Swets, J.A. (1988) Measuring the accuracy of diagnostic systems. Science, 240(4857): 1285-1293.

41. Lira, A.F., Rego, F.N. and Albuquerque, C.M. (2014) How important are environmental factors for the population structure of co-occurring scorpion species in a tropical forest? Can. J. Zool., 93(1): 15-29.

42. Ewers, R.M. and Didham, R.K. (2006) Confounding factors in the detection of species responses to habitat fragmentation. Biol. Rev. Camb. Philos. Soc., 81(1):117-142.

43. El Hidan, M.A., Touloun, O. and Boumezzough, A. (2017) Spatial relationship between environmental factors and scorpion distribution in Morocco. J. Entomol. Zool. Stud., 5(3): 674-678.

44. Sadine, S.E., Alioua, Y. and Chenchouni, H. (2012) First data on scorpion diversity and ecological distribution in the National Park of Belezma, Northeast Algeria. Serket, 13(13): 27-37.

45. Rubio, G.D. and Acosta, L.E. (2011) Geographical distribution of the space weaving spider, Chibchea salta, from northwestern Argentina: New records and bioclimatic modeling. J. Insect Sci., 11(54): 1-14.

46. El Hidan, M.A., Touloun, O., Bouazza, A., Laaradia, M.A. and Boumezzough, A. (2018) Androctonus genus species in arid regions: Ecological niche models, geographical distributions, and envenomation risk. Vet. World, 11(3): 286-292. 